\title{
Quantitative Evaluation of Segmentation- and Atlas-Based Attenuation Correction for PET/MR on Pediatric Patients
}

\author{
Ilja Bezrukov ${ }^{1,2}$, Holger Schmidt ${ }^{3}$, Sergios Gatidis ${ }^{3}$, Frédéric Mantlik ${ }^{1,2}$, Jürgen F. Schäfer ${ }^{3}$, Nina Schwenzer ${ }^{3}$, \\ and Bernd J. Pichler ${ }^{1}$ \\ ${ }^{I}$ Werner Siemens Imaging Center, Department of Preclinical Imaging and Radiopharmacy, Eberhard-Karls University Tübingen, \\ Tübingen, Germany; ${ }^{2}$ Department of Empirical Inference, Max Planck Institute for Intelligent Systems, Tübingen, Germany; and \\ ${ }^{3}$ Diagnostic and Interventional Radiology, Department of Radiology, University Hospital Tübingen, Tübingen, Germany
} Pediatric imaging is regarded as a key application for combined
PET/MR imaging systems. Because existing MR-based attenuation-
correction methods were not designed specifically for pediatric
patients, we assessed the impact of 2 potentially influential factors:
inter- and intrapatient variability of attenuation coefficients and
anatomic variability. Furthermore, we evaluated the quantification
accuracy of 3 methods for MR-based attenuation correction without
(SEGbase) and with bone prediction using an adult and a pediatric atlas (SEGwBONEad and SEGwBONEpe, respectively) on PET data of pediatric patients. Methods: The variability of attenuation coefficients between and within pediatric (5-17 y, $n=17)$ and adult (27-66 y, $n=16)$ patient collectives was assessed on volumes of interest (VOIs) in CT datasets for different tissue types. Anatomic variability was assessed on SEGwBONEad/pe attenuation maps by computing mean differences to CT-based attenuation maps for regions of bone tissue, lungs, and soft tissue. PET quantification was evaluated on VOls with physiologic uptake and on $80 \%$ isocontour VOls with elevated uptake in the thorax and abdomen/pelvis. Inter- and intrapatient variability of the bias was assessed for each VOI group and method. Results: Statistically significant differences in mean VOI Hounsfield unit values and linear attenuation coefficients between adult and pediatric collectives were found in the lungs and femur. The prediction of attenuation maps using the pediatric atlas showed a reduced error in bone tissue and better delineation of bone structure. Evaluation of PET quantification accuracy showed statistically significant mean errors in mean standardized uptake values of $-14 \% \pm 5 \%$ and $-23 \% \pm 6 \%$ in bone marrow and femur-adjacent VOls with physiologic uptake for SEGbase, which could be reduced to $0 \% \pm 4 \%$ and $-1 \% \pm 5 \%$ using SEGwBONEpe attenuation maps. Bias in soft-tissue VOls was less than $5 \%$ for all methods. Lung VOls showed high SDs in the range of $15 \%$ for all methods. For VOls with elevated uptake, mean and SD were less than 5\% except in the thorax. Conclusion: The use of a dedicated atlas for the pediatric patient collective resulted in improved attenuation map prediction in osseous regions and reduced interpatient bias variation in femuradjacent VOls. For the lungs, in which intrapatient variation was higher for the pediatric collective, a patient- or group-specific attenuation coefficient might improve attenuation map accuracy. Mean errors of $-14 \%$ and $-23 \%$ in bone marrow and femur-adjacent VOls can affect PET quantification in these regions when bone tissue is ignored.

\footnotetext{
Received Oct. 2, 2014; revision accepted Apr. 23, 2015.

For correspondence or reprints contact: Ilja Bezrukov, Department of Preclinical Imaging and Radiopharmacy, Werner Siemens Imaging Center,

Eberhard-Karls University Tübingen, Tübingen, Germany 72076.

E-mail: ilja.bezrukov@med.uni-tuebingen.de

Published online May 7, 2015.

COPYRIGHT (c) 2015 by the Society of Nuclear Medicine and Molecular Imaging, Inc.
}

Key Words: attenuation correction; PET/MR; segmentation; atlas

J Nucl Med 2015; 56:1067-1074

DOI: 10.2967/jnumed.114.149476

$\mathbf{P}$ radiation dose compared with PET/CT examinations is of particular importance for pediatric patients. The detection accuracy of malignant tumor lesions was shown to be improved for combined PET/MR imaging in comparison to PET or MR imaging alone, with increased specificity in follow-up examinations (3).

Because of the growing skeleton, anatomic variabilities are higher in the pediatric collective than in adults, possibly affecting the validity of MR-based attenuation maps, which are usually not specifically adapted for pediatric anatomy. For example, it was shown that bone density increases with increasing age (4), whereas lung density decreases (5). At present, MR-based attenuation maps in clinical routine applications of PET/MR are created by segmentation of MR images into 3-5 tissue classes and assignment of dedicated linear attenuation coefficients (LACs) to the different classes $(6,7)$. Thus, the question arises whether the attenuation maps for pediatric patients that were created with these methods designed for adult patients are of sufficient accuracy for clinical routine and whether adaptations are necessary.

The goal of this work was 3-fold: first, we wanted to address the question whether a significant age-related variability of attenuation coefficients exists between the adult and pediatric patient collectives, as such differences might produce a bias in segmentationbased attenuation maps that use predefined LACs.

Second, we wanted to find out whether the age-related anatomic variability affects the prediction accuracy of atlas-based methods. Third, we evaluated PET quantification accuracy using standard segmentation-based and atlas-based methods.

In this work, we thus systematically compared age-related variations in CT Hounsfield units (HUs) and LACs in selected volumes of interest (VOIs) between adult and pediatric CT datasets to assess the age-related variability of attenuation coefficients. The intra- and interpatient variability of LACs for both groups was also assessed. Furthermore, we compared the prediction quality of a hybrid segmentation, atlas- and pattern recognition-based method published previously (8) on the pediatric patient collective using an atlas database with adult patients versus using a newly created 
atlas database consisting of pediatric patients. The resulting attenuation maps were compared using a region-based approach. Finally, a VOI-based evaluation of the deviation of mean standardized uptake values (SUVs) from PET data corrected with the standard segmentation-based method without prediction of bone tissue (SEGbase) or the hybrid segmentation and pattern recognitionbased method with bone prediction (SEGwBONE) to PET images reconstructed using CT-based attenuation maps was performed. The intra- and interpatient variability of the bias was assessed for each VOI group and each method. PET images were reconstructed using SEGwBONE attenuation maps created with the adult atlas (SEGwBONEad) as well as the pediatric atlas (SEGwBONEpe).

\section{MATERIALS AND METHODS}

\section{Data Acquisition}

PET/CT. CT images of 17 pediatric patients (age $\pm \mathrm{SD}, 13.3 \pm 3.6 \mathrm{y}$; age range, 5-17 y; with posttransplant lymphoproliferative disorder, bronchial carcinoid, colorectal cancer, germ cell tumor, hepatocellular carcinoma $[n=2]$, lymphoma $[n=6]$, neuroendocrine tumor, pancreatic cancer, primitive neuroectodermal tumor, sarcoma, and thyroid cancer) were acquired during a PET/CT examination on a Biograph mCT (Siemens Healthcare; peak voltage, $120 \mathrm{kVp}$; tube current, $250 \mathrm{mAs}$; rotation time, $0.3 \mathrm{~s}$; collimation, $0.6 \mathrm{~mm}$; table feed, $30.7 \mathrm{~mm}$ ) with an in-plane resolution of $0.6 \times 0.6 \mathrm{~mm}$.

Additional CT images of 16 adult patients (age \pm SD, $44.6 \pm 13.0 \mathrm{y}$; range, 27-66 y; with breast cancer, colorectal cancer, fever of unknown origin, lymphoma $[n=3]$, melanoma, neuroendocrine tumor $[n=4]$, neurofibromatosis, ovarian cancer $[n=2]$, and prostate cancer $[n=2]$ ) acquired on the Biograph mCT were used for the adult patient collective.

All CT images were contrast-enhanced (portal venous phase).

All patients were enlisted in a clinical study and gave their written informed consent. For underage patients, the consent was given by their parent or guardian.

PET/MR. Corresponding PET/MR images for the pediatric patients were acquired on a Biograph mMR (Siemens). The MR images used for MR-based attenuation correction (MRAC) were acquired in breath-hold using a dual-echo spoiled gradient echo sequence with Dixon fat and water separation (echo time 1, $1.23 \mathrm{~ms}$; echo time 2, $2.46 \mathrm{~ms}$; repetition time, $3.6 \mathrm{~ms}$; flip angle, $10^{\circ}$; acquisition time, $18 \mathrm{~s}$ per bed position). No MR contrast agents were applied before the PET/MR acquisition. The MRAC sequence and additional diagnostic MR sequences were acquired parallel to the PET data acquisition, with 6 min per bed position.

A subset of 10 patients (age $\pm \mathrm{SD}, 14.5 \pm 2.27 \mathrm{y}$; range, 10-17 y) was used to create the MR/CT atlas database and evaluate the bias in atlas-based attenuation maps; of these, 9 patients (age \pm SD, $14.4 \pm$ $2.4 \mathrm{y}$; range, 10-17 y) were used for evaluation of the error in PET datasets. The PET images of these patients were acquired $103.0 \pm$ $30.4 \mathrm{~min}$ after the injection of ${ }^{18} \mathrm{~F}-\mathrm{FDG}(4.5 \pm 1.2 \mathrm{MBq} / \mathrm{kg})$.

\section{Evaluation of Age-Related LAC Variations}

An evaluation of age-related variations in $\mathrm{HU}$ values and the corresponding LACs was performed on the $33 \mathrm{CT}$ datasets, subdivided into the categories pediatric ( $n=17$; age, $13.3 \pm 3.6 \mathrm{y}$; range, $5-17 \mathrm{y}$ ) and adult $(n=16$; age, $44.6 \pm 3.0 \mathrm{y}$; range, $27-66 \mathrm{y})$ using VOI analysis.

Twenty-nine spheric VOIs were placed in each dataset such that the tissue categories muscle ( 8 VOIs), subcutaneous fat at the level of the hips (hip fat) (4 VOIs), liver (3 VOIs), lung (6 VOIs), femur (2 VOIs), and bone marrow (6 VOIs) were represented.

The mean and SD in $\mathrm{HU}$ and $\mathrm{cm}^{-1}$ were calculated for each VOI category on the adult and pediatric patient collectives. Statistical significance was tested with the Wilcoxon signed-rank test with $P$ value adjustment via the Benjamini and Hochberg method. Results were considered statistically significant for a $P$ value of less than 0.01 .

Additionally, mean and SD of all voxels from VOIs in each tissue category for each patient were computed, yielding mean patient,group

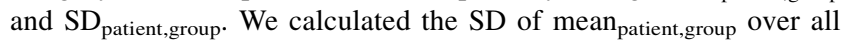
patients to assess the interpatient variability of LACs. To derive the intrapatient variability, we calculated the means of both $\mathrm{SD}_{\text {patient,group }}$ and relative $\mathrm{SD}\left(\mathrm{SD}_{\text {patient,group }} /\right.$ mean $\left._{\text {patient,group }} \times 100\right)$ over all patients.

\section{Creation of Attenuation Maps}

MR-based attenuation maps were created to evaluate the influence of age-related anatomic variability on attenuation map prediction quality and to quantify the PET bias caused by omission of osseous tissue. CT-based attenuation maps were used as a reference.

Baseline Segmentation-Based Method (SEGbase). The standard vendor-provided method for the creation of MR-based attenuation maps on the Biograph mMR was used to compute reference MR-based attenuation maps. The method segments the MR images into 5 classes, outer air, lung, fat, soft tissue, and a fat/soft tissue mix, with predefined LACs assigned to each class (6). Bone tissue is not considered as a distinct tissue class.

Segmentation-Based Method with Bone Prediction (SEGwBONE). We further evaluated the feasibility to use a previously described hybrid segmentation and atlas-based approach $(8)$ on the pediatric patient data. Segmentation-based LACs are assigned to nonosseous regions in a dataset, whereas LACs for bone areas are computed using an approach based on atlas and pattern recognition. A detailed description of the method is given in the supplemental methods (supplemental materials are available at http://jnm.snmjournals.org).

The attenuation maps were created using 2 separate atlas databases consisting of coregistered MR and CT image pairs: an adult atlas database of 11 patients, as described previously (9) (SEGwBONEad), and a newly created database of pediatric patients (SEGwBONEpe).

Creation of Pediatric Atlas Database. The newly created atlas database of pediatric patients consisted of a subset of 10 of the 17 pediatric patients (age, $14.5 \pm 2.27 \mathrm{y}$; range, 10-17 y), for which the following criteria were met: PET/MR data with bed positions including thorax, abdomen, and pelvis were available; no fat/water flip in the Dixon images occurred; the visual assessment of CT to MR registration showed no major registration artifacts. Dixon and resulting segmented attenuation map were visually inspected. None of the datasets used for the database showed an influence of the patient diseases on the attenuation maps.

All CT images of the patients in the atlas database were contrastenhanced (portal venous phase).

The CT datasets were preprocessed to remove ancillary objects such as the patient table and fixing devices. An initial alignment was performed such that the lung centers of gravity for MR and CT datasets were matched. Subsequently, the CT images were coregistered to the inphase MR data using Elastix (University Medical Center Utrecht) (10). The MR data were previously normalized for intensity variations. The registration procedure is described in the supplemental methods. Because the arm positions of the MR and CT datasets differed between acquisitions, the arm regions were substituted from the MR-based attenuation map created on the scanner, as reported previously (8).

The coregistered datasets were postprocessed to handle regions of artificially high HUs caused by the injection of contrast agent. These regions were masked automatically, checked and adjusted manually, and replaced with HU values of soft tissue. In Supplemental Figures 1A and $1 \mathrm{~B}$, respectively, the CT-based attenuation maps created from the CT before and after the removal of artificially high HUs are shown.

When using the pediatric atlas database, the attenuation maps were computed with a cross-validation approach, such that for 
each patient, 9 patients were used as atlas database to create the attenuation map.

CT-Based Attenuation Correction (CTAC). The coregistered and postprocessed CT datasets were further used to create reference CTbased attenuation maps. HU values were converted to LAC values using a piecewise bilinear transformation (11).

\section{Evaluation of Atlas Influence on Attenuation Map Prediction Quality}

To evaluate whether the anatomic variability between adult and pediatric patients affects the prediction accuracy of atlas-based attenuation maps, we applied the hybrid segmentation and atlas-based approach to compute attenuation maps for the pediatric patients using 2 different databases, an adult atlas database of 11 patients (SEGwBONEad) and the new pediatric atlas database with 10 patients (SEGwBONEpe).

A region-based evaluation was performed on the SEGbase and SEGwBONEpe/ad attenuation maps, for which the mean difference to the CT-based reference attenuation maps was computed. The following regions were used: soft tissue, bone, and lungs. Soft-tissue and lung regions were determined from the SEGbase attenuation map by assigning the tissue classes to the corresponding regions (fat and nonfat soft tissue to "soft tissue," lungs to "lungs"). Bone regions were determined from the CT-based reference attenuation maps by selecting voxels with an LAC above $0.1106 \mathrm{~cm}^{-1}$. To limit the influence of factors not related to the performance of the attenuation map prediction algorithms, the evaluated regions were further limited: regions in which the evaluation could be biased because of injection of contrast agent were excluded from the region-based evaluation, as were upper shoulder and arm regions in which also registration errors occurred due to the different arm positions in MR and CT. Furthermore, only areas that were present in all coregistered atlas datasets were used, excluding lower extremities and parts of the upper head regions from further evaluation. A sample region map is shown in Supplemental Figure 1.

The mean and SD of LAC deviations from the reference CT-based attenuation map were computed for each region category.

\section{Evaluation of PET Data}

PET datasets of 9 pediatric patients (age, $14.4 \pm 2.4 \mathrm{y}$; range, 1017 y) were reconstructed using CTAC, SEGbase, SEGwBONEad, and SEGwBONEpe attenuation maps. One patient was excluded from PET reconstruction because of the unavailability of PET raw data. VOI-based evaluations were performed using PET images reconstructed with CTAC as a reference.

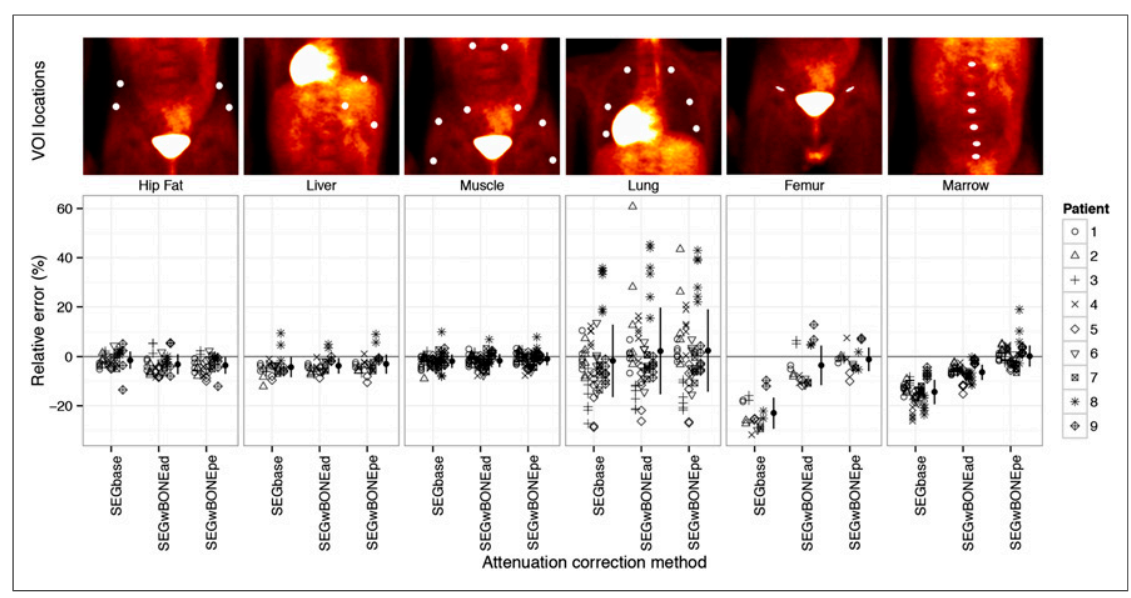

FIGURE 1. VOI positions and relative deviations of mean SUV in VOls with normal physiologic uptake for SEGbase and SEGwBONEad/pe.
Two types of VOIs were placed: spheric VOIs located in regions of normal physiologic uptake and $80 \%$ isocontour VOIs placed on regions of elevated uptake.

For VOIs on regions of normal uptake, 29 VOIs previously placed on CT images were visually adjusted side-by-side such that they were in the same positions in the PET/MR datasets, except for femur VOIs, which were placed adjacent to the bone because of low uptake in the joints.

As in the evaluation of $\mathrm{HU}$ variations, these VOIs represented the tissue types bone, muscle tissue, fat, and lung. In total, 261 VOIs were placed. The VOI placement is shown in in Figure 1.

To assess the inter- and intrapatient variability of the bias, mean and $\mathrm{SD}$ of relative differences of all voxels from VOIs in each tissue category for each patient were computed, resulting in mean patient,group,ACmethod $_{\text {, }}$ and $\mathrm{SD}_{\text {patient,group,ACmethod. The relative differences were calculated }}$ voxelwise between PET images reconstructed with MRAC and CTAC.

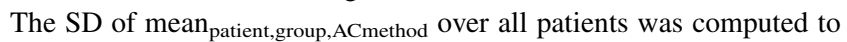
assess the interpatient variability for each method and VOI group. Intrapatient variability was derived by computing the mean over $\mathrm{SD}_{\text {patient,group,ACmethod }}$ over all patients (12).

Additionally, $80 \%$ isocontour VOIs were placed at locations of elevated uptake (lesions, urinary tract, brown adipose tissue) on PET images reconstructed with SEGbase attenuation maps. Forty-two isocontour VOIs were placed in total. The VOIs were grouped by location and proximity to osseous tissue. VOIs adjacent to bone tissue and not further away than $30 \mathrm{~mm}$ were assigned to the category near bone.

The statistical significance of the differences between VOI means in the PET images with MRAC and reference CTAC was tested with the Wilcoxon signed-rank test, with $P$ value adjustment via the Benjamini and Hochberg method. Results were considered statistically significant for a $P$ value of less than 0.01 .

Reconstruction. All PET images were reconstructed on the scanner using the manufacturer-provided implementation of 3-dimensional ordered-subset expectation maximization algorithm with 2 subsets, 21 iterations, and $4 \mathrm{~mm}$ smoothing. Automatic truncation correction of arm regions was enabled for all reconstructions.

\section{RESULTS}

\section{Evaluation of Age-Related LAC Variations}

The means, SDs, and $P$ values of the average HU values/LACs for the VOIs in CT datasets of pediatric and adult patient groups are given in Table 1, grouped by tissue categories.

Only differences in femur and lung VOIs between adult and pediatric collectives were statistically significant, with $P$ values of 0.0001 and $<0.0001$, respectively, for $\mathrm{HU}$ and $<0.0001$ and 0.0001 , respectively, for LAC.

Average HU values of lung VOIs showed a high variation within both adult and pediatric patient collectives, with an SD of $48.79 \mathrm{HU} / 0.00468 \mathrm{~cm}^{-1}$ and $76.53 \mathrm{HU} /$ $0.00735 \mathrm{~cm}^{-1}$, respectively. Intrapatient SDs were similar for the adult and pediatric patient collectives, with LACs of 0.00753 and $0.00735 \mathrm{~cm}^{-1}$, respectively; the relative intrapatient SD was higher for the adult collective, with $34.0 \%$ compared with $27.4 \%$. The interpatient SD was higher for the pediatric collective, with 0.00689 $\mathrm{cm}^{-1}$ compared with $0.00359 \mathrm{~cm}^{-1}$ for the adult collective.

Although the differences for bone marrow VOIs were not statistically significant with 
$P=0.0703$ for HU and $P=0.0586$ for LAC, the pediatric collective showed a higher SD of $44.82 \mathrm{HU} / 0.00229 \mathrm{~cm}^{-1}$, compared with $30.72 \mathrm{HU} / 0.00157 \mathrm{~cm}^{-1}$ for the adult collective. A higher SD for the pediatric patient collective was also observed in the hip fat VOIs.

Intrapatient absolute and relative SDs averaged over each patient for soft-tissue VOI categories were similar for both patient collectives in the range of $0.0011-0.0 .00187 \mathrm{~cm}^{-1} / 1.1 \%-$ 2.1\% (Supplemental Table 1).

\section{Atlas-Related Fidelity of Attenuation Maps}

The prediction errors of MR-based attenuation maps that were created using an atlas of pediatric or adult patients are shown in Figure 2. The bias is similar for both methods in nonosseous tissue. For osseous tissue, SEGwBONEpe attenuation maps show a smaller deviation from CT attenuation maps than SEGwBONEad. Visually, the attenuation maps created with the pediatric atlas database show a better delineation of bony structures. In Figure 3, a comparison of attenuation maps created using the 2 different atlas databases is shown for a representative patient. Here, a better delineation of vertebrae is clearly notable. Registration-related artifacts occur in attenuation maps created with both atlases, particularly in the shoulder areas due to the mismatch of arms positions between MR and CT images.

\section{Quantitative Evaluation of PET Data}

VOIs with Physiologic Uptake. The relative differences of the VOIs with physiologic uptake in PET images reconstructed with SEGbase or SEGwBONEad/pe and PET images reconstructed with reference CTAC are shown in Figure 1. Additionally, absolute differences, minimum and maximum deviations, and the $P$ values are shown in Table 2 . The corresponding scatterplots of mean activity in the VOIs are shown in Supplemental Figure 2. The regression fit parameters are given in Supplemental Table 2. Voxelwise means and SDs of relative differences for all voxels in each VOI group averaged over each patient are given in Supplemental Table 3 and Supplemental Figure 3.

VOIs located in soft-tissue regions (hip fat, liver, and muscle) show minor mean deviations with both mean SUV and SD below $5 \%$ for all methods. The deviations of liver and muscle tissue VOIs for SEGbase; of hip fat and liver VOIs for SEGwBONEpe; and of hip fat, liver, and muscle VOIs for SEGwBONEad were statistically significant.
The scatterplots for soft-tissue VOIs also show a good agreement with reference CTAC, with $R^{2}$ values greater than 0.99 for all methods.

Averaged intrapatient SDs of the relative error for soft-tissue VOI categories were below 5\% for all methods.

The differences to CTAC for lung VOIs exhibited a high variability, compared with soft-tissue VOIs. Although the nonsignificant mean deviations are below $5 \%$ for all methods, the SD range was from $15 \%$ to $18 \%$. This is reflected by the lower $R^{2}$ values of $0.774,0.722$, and 0.675 for SEGbase, SEGwBONEpe, and SEGwBONEad, respectively. The interpatient SDs were higher than intrapatient SDs, with a range of $14 \%-15 \%$ for the former and $7 \%-8 \%$ for the latter.

VOIs placed adjacent to femur joints exhibited the highest relative and absolute mean deviations for the SEGbase method (where bone is not predicted), showing a relative error of $-23.0 \% \pm$ 6.4\%. SEGwBONEpe showed a good agreement with reference CTAC, with a relative deviation of $-1.1 \% \pm 4.8 \%$. SEGwBONEad showed a slightly higher mean error and higher SD of $-3.6 \% \pm$ $8.1 \%$. The difference for SEGbase was statistically significant, with a $P$ value of less than 0.0001 . The $R^{2}$ values were 0.920 , 0.967, and 0.924 for SEGbase, SEGwBONEpe, and SEGwBONEad, respectively. Interpatient SD was lowest for SEGwBONEpe with $4.4 \%$, whereas SEGwBONEad and SEGbase exhibited $6.4 \%$ and $8.2 \%$, respectively. Intrapatient SDs were below $2.1 \%$ for all methods.

VOIs located inside bone marrow showed an error of $-14.4 \% \pm$ $5.0 \%, 0.3 \% \pm 4.3 \%$, and $-6.4 \% \pm 3.2 \%$ for SEGbase, SEGwBONEpe, and SEGwBONEad, respectively. The bias for SEGbase and SEGwBONEad was statistically significant, with a $P$ value of less than 0.0001 . The $R^{2}$ value was on a similar scale with 0.990 for SEGbase/SEGwBONEpe and 0.992 for SEGwBONEad. Interpatient SDs were $4.9 \%, 3.3 \%$, and $2.6 \%$ for SEGbase, SEGwBONEpe, and SEGwBONEad, respectively. Intrapatient SDs were below $2.1 \%$ for all methods.

VOIs with Elevated Uptake. Relative differences of mean activity for VOIs in regions of elevated uptake are shown in Figure 4. The differences are grouped by their anatomic location and proximity to osseous tissue. Furthermore, absolute differences, minimum and maximum deviations, and $P$ values are shown in Table 3 .

Both MRAC methods with and without bone prediction showed similar results with only minor differences, except in the thorax, in which also the largest mean deviations were found. For thoracic

TABLE 1

Comparison of Mean HU Values and LACs for Pediatric $(n=17)$ and Adult $(n=16)$ Patient Collectives

\begin{tabular}{|c|c|c|c|}
\hline \multirow[b]{2}{*}{ VOI } & \multicolumn{2}{|c|}{ Mean $\pm \mathrm{SD}\left(\mathrm{HU}, \mathrm{cm}^{-1} \times 10^{4}\right)$} & \multirow[b]{2}{*}{$P$} \\
\hline & Pediatric & Adult & \\
\hline Hip fat (4 VOls/patient) & $-93.50 \pm 27.81(870.23 \pm 26.60)$ & $-101.62 \pm 11.77(862.44 \pm 11.30)$ & $0.2189(0.1642)$ \\
\hline Liver (3 VOls/patient) & $111.87 \pm 28.71(1,037.80 \pm 14.92)$ & $107.63 \pm 26.83(1,035.19 \pm 15.99)$ & $0.9804(0.9804)$ \\
\hline Muscle (8 VOls/patient) & $58.35 \pm 8.60(1,008.98 \pm 5.58)$ & $57.28 \pm 7.89(1,008.64 \pm 5.21)$ & $0.4272(0.6397)$ \\
\hline Lung (6 VOls/patient) & $-706.32 \pm 76.53(281.92 \pm 73.47)$ & $-769.04 \pm 48.79(221.71 \pm 46.82)$ & $<0.0001^{\star}\left(<0.0001^{\star}\right)$ \\
\hline Femur (2 VOIs/patient) & $309.99 \pm 34.98(1,139.11 \pm 17.84)$ & $351.34 \pm 33.39(1,160.19 \pm 17.03)$ & $0.0001^{\star}\left(<0.0001^{\star}\right)$ \\
\hline Marrow (6 VOls/patient) & $189.44 \pm 44.82(1,077.59 \pm 22.91)$ & $196.34 \pm 30.72(1,081.14 \pm 15.68)$ & $0.0703(0.0586)$ \\
\hline
\end{tabular}




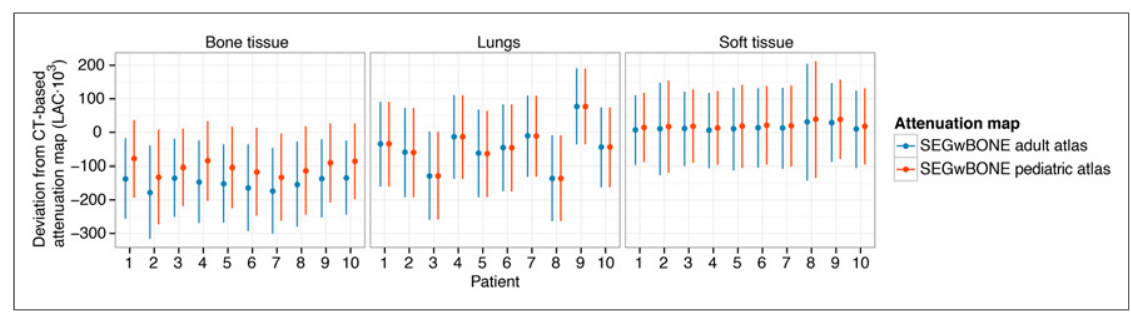

FIGURE 2. Prediction errors for MR-based attenuation maps created with the SEGwBONE method for 2 different atlas databases, pediatric and adult, using CT-based attenuation maps as reference.

VOIs not located in bone proximity SEGbase exhibited a deviation of $-6.7 \% \pm 5.4 \%$ versus $-0.7 \% \pm 4.4 \%$ and $0.2 \% \pm 7.4 \%$ for SEGwBONEpe and SEGwBONEad. For thoracic VOIs in bone proximity, SEGbase showed an underestimation of $-2.4 \% \pm$ $6.5 \%$, whereas SEGwBONEpe and SEGwBONEad showed an overestimation of $5.6 \% \pm 5.2 \%$ and $2.3 \% \pm 3.9 \%$.

For the abdomen/pelvis region, mean differences below 5\% in comparison to reference CTAC for all MRAC methods were observed.

\section{DISCUSSION}

Our findings did not reveal statistically significant differences for soft-tissue VOIs in CT datasets between pediatric and adult patient collectives. It is thus reasonable to expect that no specific adaptation of soft-tissue LACs to assign to segmented tissue regions in segmentation-based MRAC is required for pediatric patients.

Lung density exhibits a high variability between patients, such that the assignment of nonpatient-specific lung LACs can affect PET quantification $(7,9,13)$. In this study, high variations of average HU values in lung VOIs were observed between the pediatric and adult collectives and within each population. These variations between the collectives are partially age-dependent, because lung density correlates with lung volume (14), and the lung volume increases during child growth. A linear decrease of mean lung density in CT was observed by Vock et al. for children aged 7-18 y (15). The amount of variation within the collectives is likely

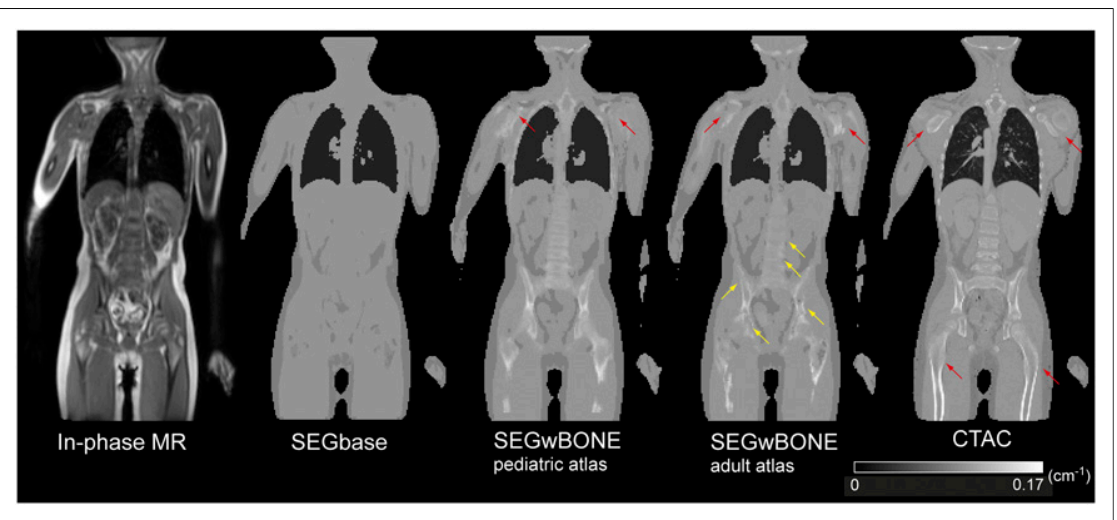

FIGURE 3. Sample slice of in-phase MR image, attenuation maps from standard segmentation (SEGbase) and predicted using 2 different atlas databases (pediatric and adult), and corresponding reference CT-based attenuation map. Red arrows denote artifacts in predicted and reference attenuation maps, which are related to registration. Yellow arrows denote regions in which bone structure fidelity is affected. caused by variations of lung density between the patients and intrapatient variations, which can be attributed to density gradients in the ventral-dorsal and apicalbasal directions. The observed amount of intrapatient variation was slightly higher for the pediatric collective in our study, with a relative SD of $34 \%$ compared with $27 \%$. The observed variation is reflected in the differences for lung VOIs with physiologic uptake in PET, where an SD in the range of $15 \%$ for both MRAC methods was observed. Although the correlation between lung volume and density could be used to determine patient-specific or age-specific LACs, a uniform lung LAC would not perform as well as an approach that predicts LACs for individual voxels or slices, as was observed by Marshall et al. (16). Therefore, approaches based on specific MR sequences (16) are potentially more robust but would add additional MR scanning time.

The average LACs in femur bone VOIs for the pediatric and adult patient collectives exhibited a statistically significant increase for the adult patient collective, which could potentially affect PET quantification results. A probable cause for the increase is an age-related compression of the joint bones due to continuous load.

Because the mean LACs of bone marrow VOIs were not significantly different between the adult and pediatric collectives, the PET quantification results are likely not to be affected by agerelated LAC differences to a clinically relevant degree, although the amount of interpatient variation was higher for the pediatric than for the adult collective.

The use of a dedicated atlas for pediatric patients improved the prediction quality of the attenuation maps in bone tissue areas, with a better delineation of bone structures. The improved prediction quality is to a large part due to improved atlas database registration results, given the lower anatomic variations between pediatric patients compared with variations between pediatric and adult patients. A better match of bone densities of the pediatric atlas database, as was found in the femur, might also contribute. An improvement in quantification accuracy is likely to be expected in osseous lesions or lesions adjacent to bone. Because the created attenuation maps use the same segmentationbased results in nonosseous regions, independent of the atlas, the differences in the lungs and soft tissue were similar using both atlases.

The selection of matching patients for an atlas-based prediction based on age, patient height, or body mass index might thus be a viable way to improve robustness and prediction quality of atlas-based attenuation maps.

In agreement with the small differences in mean HU values of soft-tissue VOIs between the adult and pediatric collectives found in CT data, SUVs from PET VOIs with normal physiologic uptake revealed only minor deviations, with mean and SD below 5\% for SEGbase and SEGwBONEpe/ad in muscle, hip fat, and liver. The mean absolute differences of these VOIs were below 0.05 SUV; thus, statistical significance was not reached for all regions. 
TABLE 2

Relative Differences (\%) and Absolute Differences (SUV) on VOls in Physiologic Uptake*

\begin{tabular}{|c|c|c|c|c|c|c|c|}
\hline \multirow[b]{2}{*}{ VOI } & \multirow[b]{2}{*}{$n$} & \multirow[b]{2}{*}{ AC method } & \multicolumn{3}{|c|}{ Relative difference (\%) } & \multirow[b]{2}{*}{ Absolute difference (SUV) } & \multirow[b]{2}{*}{$P$} \\
\hline & & & Mean \pm SD & Minimum & Maximum & & \\
\hline Hip fat & 36 & SEGbase & $-1.41 \pm 3.52$ & -13.51 & 5.23 & $-0.00 \pm 0.01$ & 0.2134 \\
\hline Hip fat & 36 & SEGwBONEpe & $-3.58 \pm 3.43$ & -12.07 & 2.41 & $-0.01 \pm 0.01$ & $0.0021^{*}$ \\
\hline Hip fat & 36 & SEGwBONEad & $-3.09 \pm 4.06$ & -8.53 & 5.61 & $-0.01 \pm 0.01$ & $0.0001^{*}$ \\
\hline Liver & 27 & SEGbase & $-4.25 \pm 4.12$ & -12.15 & 9.53 & $-0.05 \pm 0.10$ & $0.0021^{*}$ \\
\hline Liver & 27 & SEGwBONEpe & $-3.05 \pm 3.90$ & -10.52 & 9.10 & $-0.03 \pm 0.09$ & $0.0031^{*}$ \\
\hline Liver & 27 & SEGwBONEad & $-3.72 \pm 3.19$ & -8.72 & 5.04 & $-0.05 \pm 0.07$ & $0.0032^{*}$ \\
\hline Muscle & 72 & SEGbase & $-1.85 \pm 2.75$ & -8.98 & 10.07 & $-0.01 \pm 0.02$ & $<0.0001^{*}$ \\
\hline Muscle & 72 & SEGwBONEpe & $-0.88 \pm 2.66$ & -7.70 & 7.95 & $-0.005 \pm 0.02$ & 0.0309 \\
\hline Muscle & 72 & SEGwBONEad & $-1.59 \pm 2.67$ & -7.79 & 6.98 & $-0.01 \pm 0.02$ & $<0.0001^{*}$ \\
\hline Lung & 54 & SEGbase & $-1.73 \pm 14.75$ & -28.79 & 35.79 & $-0.01 \pm 0.04$ & 0.0947 \\
\hline Lung & 54 & SEGwBONEpe & $2.36 \pm 16.64$ & -26.99 & 43.43 & $0.00 \pm 0.05$ & 0.9590 \\
\hline Lung & 54 & SEGwBONEad & $2.20 \pm 17.54$ & -26.28 & 60.79 & $0.00 \pm 0.05$ & 0.9590 \\
\hline Femur & 18 & SEGbase & $-23.01 \pm 6.35$ & -31.77 & -9.62 & $-0.24 \pm 0.09$ & $0.0006^{*}$ \\
\hline Femur & 18 & SEGwBONEpe & $-1.10 \pm 4.80$ & -10.01 & 7.61 & $-0.01 \pm 0.05$ & 0.2136 \\
\hline Femur & 18 & SEGwBONEad & $-3.56 \pm 8.06$ & -11.84 & 12.97 & $-0.04 \pm 0.08$ & 0.0518 \\
\hline Marrow & 54 & SEGbase & $-14.42 \pm 4.96$ & -26.16 & -4.30 & $-0.19 \pm 0.06$ & $<0.0001^{*}$ \\
\hline Marrow & 54 & SEGwBONEpe & $0.25 \pm 4.30$ & -6.43 & 18.99 & $-0.00 \pm 0.05$ & 0.9590 \\
\hline Marrow & 54 & SEGwBONEad & $-6.41 \pm 3.12$ & -15.18 & -0.25 & $-0.09 \pm 0.05$ & $<0.0001^{*}$ \\
\hline
\end{tabular}

*Statistically significant differences.

Differences are averaged over all evaluated patients.

Similar error magnitudes below $5 \%$ were reported by Keereman et al. for soft-tissue regions of segmentation-based attenuation maps in a PET simulation study (13) as well as by Bezrukov et al. (8) for lesions in soft tissue. Thus, previous findings in adults that segmentation-based methods without bone prediction are sufficient for quantitative PET imaging in soft-tissue regions hold true for pediatric patients.

We observed a high amount of variation for lung VOIs with physiologic uptake in PET, with an SD in the range of $15 \%$ and markedly lower $R^{2}$ values of $0.774,0.722$, and 0.675 for SEGbase and SEGwBONEpe, and SEGwBONEad, respectively, when compared with other tissues. The better performance of SEGbase is most likely due to the observation that the patient with the highest deviations shows a particularly low lung density of $-862 \mathrm{HU}$ or a LAC of $0.0132 \mathrm{~cm}^{-1}$, which is closer to the predefined LAC of SEGbase $\left(0.0224 \mathrm{~cm}^{-1}\right)$ than to the lung LAC of SEGwBONE $\left(0.024 \mathrm{~cm}^{-1}\right)$. Although the relative differences in the lungs exceed $40 \%$ (Fig. 1), the physiologic activity is low, such that absolute differences are not as pronounced with an SD of 0.04 SUV and 0.05 SUV for SEGbase and SEGwBONEpe/ad, respectively. An assignment of nonmatching LACs for lung tissue could potentially affect lung lesions or lesions in the thoracic region. In the case described above, in which the mismatch between assigned lung density and assigned lung LACs is particularly large, a lesion located in the thorax was overestimated by $9.1 \%$ and $14.2 \%$ for SEGbase and SEGwBONEpe, respectively, which could partially be attributed to the mismatch in lung densities.

Although the mean SUV underestimation for SEGbase was highest $(-23.0 \% \pm 6.4 \%)$ in VOIs with physiologic uptake located directly adjacent to femoral bone, the magnitude is comparable to or slightly lower than the results of a similar evaluation on VOIs with physiologic uptake, for which mean underestimations for pelvic

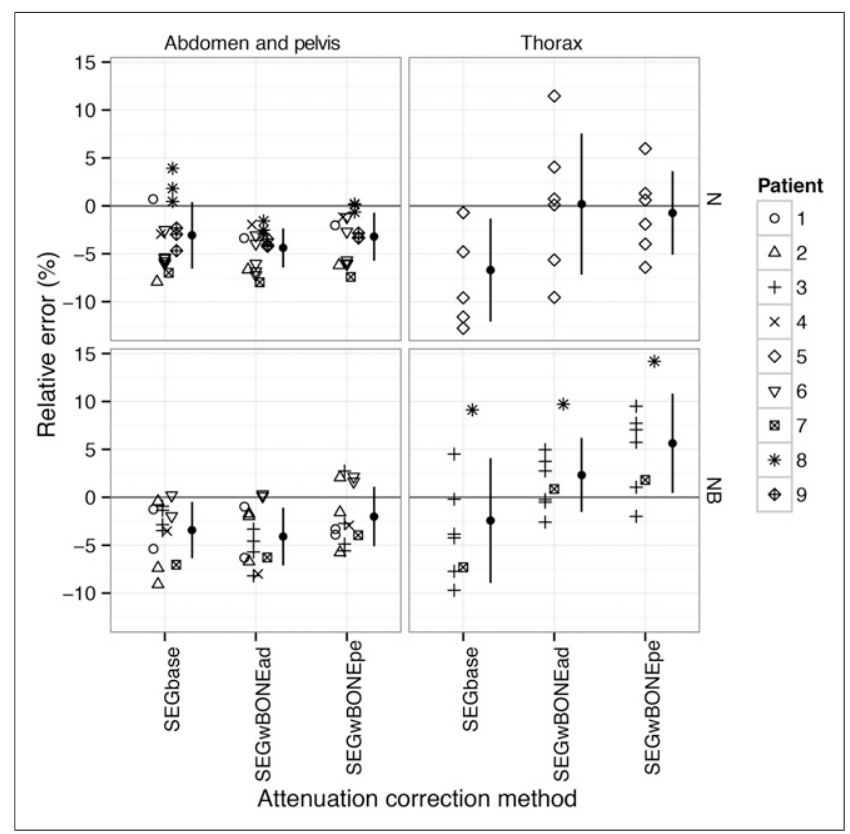

FIGURE 4. Relative deviations in mean SUV in VOls with elevated uptake in tissue categories nonosseous tissue (N) and near bone (NB). 
TABLE 3

Relative Differences (\%) and Absolute Differences (SUV) on Elevated Uptake VOls

\begin{tabular}{|c|c|c|c|c|c|c|c|c|}
\hline \multirow[b]{2}{*}{ Location } & \multirow[b]{2}{*}{ Tissue } & \multirow[b]{2}{*}{$n$} & \multirow[b]{2}{*}{ AC method } & \multicolumn{3}{|c|}{ Relative difference (\%) } & \multirow[b]{2}{*}{ Absolute difference (SUV) } & \multirow[b]{2}{*}{$P$} \\
\hline & & & & Mean \pm SD & Minimum & Maximum & & \\
\hline $\mathrm{AbPe}$ & 15 & $\mathrm{~N}$ & SEGbase & $-3.07 \pm 3.47$ & -7.92 & 3.91 & $-0.09 \pm 0.49$ & 0.1808 \\
\hline $\mathrm{AbPe}$ & 15 & $\mathrm{~N}$ & SEGwBONEpe & $-3.22 \pm 2.51$ & -7.42 & 0.20 & $-0.19 \pm 0.27$ & $0.0034^{*}$ \\
\hline $\mathrm{AbPe}$ & 15 & $\mathrm{~N}$ & SEGwBONEad & $-4.39 \pm 2.04$ & -7.98 & -1.57 & $-0.35 \pm 0.30$ & $0.0007^{*}$ \\
\hline $\mathrm{AbPe}$ & 13 & NB & SEGbase & $-3.42 \pm 2.95$ & -9.08 & 0.21 & $-0.12 \pm 0.11$ & $0.0029^{*}$ \\
\hline $\mathrm{AbPe}$ & 13 & NB & SEGwBONEpe & $-2.00 \pm 3.10$ & -5.75 & 2.74 & $-0.08 \pm 0.14$ & 0.1377 \\
\hline $\mathrm{AbPe}$ & 13 & NB & SEGwBONEad & $-4.10 \pm 3.02$ & -8.20 & 0.32 & $-0.18 \pm 0.16$ & $0.0037^{\star}$ \\
\hline Th & 6 & $\mathrm{~N}$ & SEGbase & $-6.70 \pm 5.38$ & -12.78 & -0.70 & $-0.26 \pm 0.24$ & 0.0750 \\
\hline Th & 6 & $\mathrm{~N}$ & SEGwBONEpe & $-0.74 \pm 4.36$ & -6.42 & 5.98 & $-0.05 \pm 0.16$ & 0.7500 \\
\hline Th & 6 & $\mathrm{~N}$ & SEGwBONEad & $0.19 \pm 7.37$ & -9.56 & 11.46 & $-0.04 \pm 0.26$ & 1.0000 \\
\hline Th & 8 & NB & SEGbase & $-2.42 \pm 6.51$ & -9.70 & 9.12 & $0.18 \pm 0.97$ & 0.5531 \\
\hline Th & 8 & NB & SEGwBONEpe & $5.63 \pm 5.19$ & -1.99 & 14.19 & $0.64 \pm 1.34$ & 0.0781 \\
\hline Th & 8 & NB & SEGwBONEad & $2.34 \pm 3.87$ & -2.59 & 9.71 & $0.39 \pm 0.97$ & 0.2604 \\
\hline
\end{tabular}

*Statistically significant differences.

VOls are placed in abdomen/pelvis $(\mathrm{AbPe})$ and thorax $(\mathrm{Th})$ at locations in normal tissue $(\mathrm{N})$ and near bone (NB) averaged over all evaluated patients.

VOIs in osseous tissue between $-31 \%$ and $-17 \%$ were reported (9). An underestimation for bone regions of $30.1 \% \pm 27.5 \%$ was reported from a comparison study of PET/CT datasets with PET/MR datasets of the same patient with a mean age of $58 \mathrm{y}$ (17). Although not directly comparable, as a variety of different bone regions was evaluated and PET images from 2 different systems were used, the reported underestimation is higher than the bias in our study. The lower average HU values of femoral bone VOIs for the pediatric collective, combined with the smaller size of bones and thus a smaller amount of high-attenuating bone tissue along the lines of response, suggests a smaller impact on PET quantification compared with adult patients, if bone prediction in attenuation maps is omitted.

The mean underestimation of $-14 \%$ that was observed for bone marrow VOIs when bone was not predicted needs to be considered when quantitative PET readings are performed. Because the characterization of bone marrow is of particular interest in pediatric patients (e.g., higher incidence of osteosarcoma and Ewing sarcoma, which are primary bone tumors and also tend to spread to the bone), the application of an MRAC method that predicts bone tissue would improve correspondence with PET/CT data and quantification accuracy. If an atlas-based method such as SEGwBONE is used, the accuracy can benefit from the use of a dedicated pediatric atlas, as it can provide better atlas registration results and, as seen in the example of femur bone, more accurate density estimations.

Both intra- and interpatient variability contribute to the magnitude of the bias SD (12). We observed a higher amount of interpatient SD than intrapatient SD for all VOI categories in our study except hip fat and muscle, for which interpatient SD was slightly lower, but both inter- and intrapatient SDs were below $3 \%$. The intrapatient SD was below 3\% for all regions except the lungs, for which it was in the range of $7 \%$ for all $\mathrm{AC}$ methods. In comparison, interpatient SD for the lungs was in the range of $15 \%$. Ouyang et al. reported almost equal contributions of inter- and intrapatient lung density variations in the range of $10 \%$ to the overall lung SD (12). The higher amount of interpatient lung bias variation in our study is likely to be caused by the amount of interpatient SD in lung LACs, which was higher in the pediatric collective $\left(69 \mathrm{~cm}^{-1} \times 10^{4}\right)$ than in the adult collective $\left(36 \mathrm{~cm}^{-1} \times 10^{4}\right)$.

VOIs of PET-avid lesions not located in the thorax showed minor deviations from reference PET images corrected with CTAC for both methods, suggesting that for most lesions in clinical routine the PET quantification on pediatric patients can be performed using existing attenuation map algorithms with only small adaptations. Particularly, an adaptation of LACs for segmentation-based algorithms is not necessary for soft-tissue classes. However, lesions in the thorax would potentially benefit from an adapted lung LAC, as discussed in the previous paragraph. For VOIs with elevated uptake, the underestimation for SEGbase in VOIs near osseous tissue is smaller than for VOIs further away from bone, which is likely to be attributed to the differences in lung LACs for the patients in question.

The study has some limitations that need to be considered. First, the reference attenuation maps used in this study were created from corresponding CT datasets that were coregistered to the MR images of the same patients. Remaining residual inaccuracies in the registration can affect the results. The different arm positions that required postprocessing could cause a potentially lower bias, particularly in the thoracic VOIs.

Second, the presence of intravascular CT contrast agent is known to cause PET artifacts in thoracic veins containing the undiluted agent (18). As we replaced the HU values of affected regions with $\mathrm{HU}$ values of soft tissue, the effect is not likely to cause a systematic bias in the evaluation, as other studies indicate that the presence of intravascular contrast agents at normal concentrations has little effect on PET/CT quantification accuracy $(19,20)$. However, contrast-enhanced CTs are used for AC in $\mathrm{PET} / \mathrm{CT}$ in clinical routine at our hospital, and the contrast agent 
was present in all patients of this study. Also, we used VOIs with physiologic activity to assess the quantification accuracy. Because the activity and the tissue composition of tumor tissue can vary (21), VOIs with physiologic activity may not always show errors consistent with lesion VOIs.

Finally, additional variability of bone density may be introduced through treatment side effects, for example, caused by corticosteroids during chemotherapy. The chosen cohort is representative for patients examined in PET/CT and PET/MR, which might be during or shortly after therapy. However, an evaluation of a potential impact on PET quantification would require a larger patient collective.

We did not consider toddlers in our study, thus our conclusions do not extend to very young patients. For these patients, it is reasonable to expect that a patient-specific lung LAC might become more important, because the lung density would further increase (5). Although the anatomic variability might require the use of a dedicated toddler atlas if atlas-based bone AC is desired, the bias by leaving bone attenuation out might further decrease, because the smaller bone size would lead to a smaller impact on PET quantification. Thus, dependent on the accuracy requirements, bone AC might be not necessary for toddlers; however, this issue should be further evaluated.

\section{CONCLUSION}

We investigated the accuracy of MR-based attenuation maps for PET quantification in pediatric patients in simultaneous PET/MR. To this end, we evaluated whether age-related variabilities in tissue density and anatomy affect the accuracy of MR-based attenuation maps.

Statistically significant variations in CT density between adult and pediatric collectives were found in lung and femur VOIs. The use of a dedicated atlas for the pediatric patient collective resulted in an improved attenuation map prediction in osseous regions. For the lungs, for which the intrapatient variation was higher for the pediatric collective, a patient- or group-specific LAC could improve the accuracy of attenuation maps.

Subsequent evaluation of PET quantification accuracy using 2 MRAC methods-SEGbase, which does not account for bone, and SEGwBONE, which predicts bone tissue using the adult or pediatric atlas database and pattern recognition-yielded statistically significant mean errors of $-14 \%$ and $-23 \%$ in bone marrow and femur-adjacent VOIs with physiologic uptake for SEGbase, a magnitude that can affect PET quantification. SEGwBONEad showed mean errors of $-6 \%$ and $4 \%$, which could be further reduced to $0.3 \%$ and $-1 \%$ with SEGwBONEpe. Interpatient bias variation in femur-adjacent VOIs was reduced for SEGwBONEpe, with $4.4 \%$ in comparison to $8.2 \%$ for SEGwBONEad.

Overall, SUV underestimations in pediatric patients near or inside bony structures are less prominent than in adults. Nevertheless, for quantitative evaluation of primary bone tumors, having a higher incidence in children, consideration of bone (e.g., by atlas-based approaches) is still important. Especially for small children, the increased lung density might be important to be considered.

\section{DISCLOSURE}

The costs of publication of this article were defrayed in part by the payment of page charges. Therefore, and solely to indicate this fact, this article is hereby marked "advertisement" in accordance with 18 USC section 1734. Financial research support was provided through "Deutsche Forschungsgemeinschaft" grants PI771/ 5-1 and PI771/1-1 and through the Swiss Werner Siemens Foundation. No other potential conflict of interest relevant to this article was reported.

\section{REFERENCES}

1. Bailey DL, Barthel H, Beyer T, et al. Summary report of the first international workshop on PET/MR imaging, March 19-23, 2012, Tübingen, Germany. Mol Imaging Biol. 2013;15:361-371.

2. Schäfer JF, Gatidis S, Schmidt H, et al. Simultaneous whole-body PET/MR imaging in comparison to PET/CT in pediatric oncology: initial results. Radiology. 2014;273:220-231.

3. Pfluger T, Melzer HI, Mueller WP, et al. Diagnostic value of combined ${ }^{18} \mathrm{~F}-\mathrm{FDG}$ PET/MRI for staging and restaging in paediatric oncology. Eur J Nucl Med Mol Imaging. 2012;39:1745-1755.

4. McKay HA, Petit M, Bailey D, Wallace WM, Schutz RW, Khan KM. Analysis of proximal femur DXA scans in growing children: comparisons of different protocols for cross-sectional 8-month and 7-year longitudinal data. J Bone Miner Res. 2000;15:1181-1188.

5. Long FR, Williams RS, Castile RG. Inspiratory and expiratory CT lung density in infants and young children. Pediatr Radiol. 2005;35:677-683.

6. Martinez-Möller A, Souvatzoglou M, Delso G, et al. Tissue classification as a potential approach for attenuation correction in whole-body PET/MRI: evaluation with PET/CT data. J Nucl Med. 2009;50:520-526.

7. Schulz V, Torres-Espallardo I, Renisch S, et al. Automatic, three-segment, MRbased attenuation correction for whole-body PET/MR data. Eur J Nucl Med Mol Imaging. 2011;38:138-152.

8. Bezrukov I, Schmidt H, Mantlik F, et al. MR-based attenuation correction methods for improved PET quantification in lesions within bone and susceptibility artifact regions. J Nucl Med. 2013;54:1768-1774.

9. Hofmann M, Bezrukov I, Mantlik F, et al. MRI-based attenuation correction for whole-body PET/MRI: quantitative evaluation of segmentation- and atlas-based methods. J Nucl Med. 2011;52:1392-1399.

10. Klein S, Staring M, Murphy K, Viergever M, Pluim JPW. Elastix: a toolbox for intensity-based medical image registration. IEEE Trans Med Imaging. 2010;29: 196-205.

11. Carney JPJ, Townsend DW, Rappoport V, Bendriem B. Method for transforming CT images for attenuation correction in PET/CT imaging. Med Phys. 2006;33: 976-983.

12. Ouyang J, Chun S, Petibon Y, Bonab A. Bias atlases for segmentation-based PET attenuation correction using PET-CT and MR. IEEE Trans Nucl Sci. 2013;60: 3373-3382.

13. Keereman V, Van Holen R, Mollet P, Vandenberghe S. The effect of errors in segmented attenuation maps on PET quantification. Med Phys. 2011;38:60106019 .

14. Gould GA, Redpath T, Ryan M, et al. Lung CT density correlates with measurements of airflow limitation and the diffusing capacity. Eur Respir J. 1991;4:141146.

15. Vock P, Malanowski D, Tschaeppeler H, Kirks DR, Hedlund LW, Effmann EL. Computed tomographic lung density in children. Invest Radiol. 1987;22:627631.

16. Marshall HR, Prato FS, Deans L, Théberge J, Thompson RT, Stodilka RZ. Variable lung density consideration in attenuation correction of whole-body PET/ MRI. J Nucl Med. 2012;53:977-984.

17. Eiber M, Takei T, Souvatzoglou M, et al. Performance of whole-body integrated ${ }^{18} \mathrm{~F}-\mathrm{FDG}$ PET/MR in comparison to PET/CT for evaluation of malignant bone lesions. J Nucl Med. 2014;55:191-197.

18. Antoch G, Freudenberg LS, Egelhof T, et al. Focal tracer uptake: a potential artifact in contrast-enhanced dual-modality PET/CT scans. J Nucl Med. 2002;43: 1339-1342.

19. Yau Y-Y, Chan W-S, Tam Y-M, et al. Application of intravenous contrast in PET/ CT: does it really introduce significant attenuation correction error? J Nucl Med. 2005;46:283-291.

20. Beyer T, Antoch G, Bockisch A, Stattaus J. Optimized intravenous contrast administration for diagnostic whole-body ${ }^{18}$ F-FDG PET/CT. J Nucl Med. 2005;46: $429-435$.

21. Samarin A, Burger C, Wollenweber SD, et al. PET/MR imaging of bone lesions: implications for PET quantification from imperfect attenuation correction. Eur J Nucl Med Mol Imaging. 2012;39:1154-1160. 